\title{
Forma familiar de la enfermedad de Creutzfeldt-Jakob: marcadores genéticos en 4 familias chilenas
}

\author{
Luis Cartier $\mathbf{R}^{1}$, Jorge Fernández $\mathbf{0}^{2}$, Eugenio Ramírez $\mathbf{V}^{3,4}$. \\ Genetic markers in four \\ Chilean families with familial \\ Creutfeldt-Jakob disease
}

Background: Creutzfeldt-Jakob disease (CJD) is a form of transmissible spongiform encephalopathy, in which a prion protein $(\mathrm{PrPSC})$ accumulates in the brain of affected individuals. Chile has a prevalence of CJD that is more than twice than in the rest of the world and has the highest rate of familial forms. These later forms are associated with the heterozygocity of codon 200 of PrP protein gene. Aim: To search susceptibility genetic markers of CJD in members of families affected by CJD. Material and methods: A blood sample was obtained from 50 individuals pertaining to four families affected by CJD. DNA from peripheral mononuclear cells was amplified by polymerase chain reaction and sequenced for the gene that codifies PrP protein. Results: In family A, 21 of 23 members were homozygotes for codon 129 (Met/Met) and eight were simultaneously heterozygotes for codon 200 (Glu/Lys). In family B, six of nine members were homozygotes for codon 129, five were heterozygotes for codon 200 and four had both mutations. In family C, the four analyzed subjects were homozygotes for codon 129 and two were simultaneously heterozygotes for codon 200. In family D, nine of 14 members were homozygotes for codon 129 and two were simultaneously homozygotes for codon 200 . No family had polymorphisms for codon 219. Conclusions: Thirty two percent of analyzed subjects were homozygotes for codon 129 and heterozygotes for codon 200, condition that defines the genetic susceptibility to acquire CJD. The dominant tendency of these genotypes could explain the higher incidence of CJF in Chile (Rev Méd Chile 2006; 134: 1116-22).

(Key w ords: CJD (Creutzfeldt-Jakob disease); Gene components; PrPSc proteins)

\footnotetext{
Recibido el 30 de octubre, 2005. Aceptado el 17 de marzo, 2006.

${ }^{1}$ Departamento de Ciencias Neurológicas, Facultad de Medicina, Universidad de Chile, Hospital del Salvador, Santiago, Chile. ${ }^{2}$ Sección de Biología Molecular y ${ }^{3}$ Departamento de Virología, Instituto de Salud Pública de Chile. ${ }^{4}$ Programa de Virología, Instituto de Ciencias Biomédicas, Facultad de Medicina, Universidad de Chile.
}

L a enfermedad Creutzfeldt-Jakob (ECJ) pertenece al grupo de las encefalopatías espongiformes transmisibles (EET) que afecta a diversos mamíferos, entre ellos a los seres humanos. Las formas humanas de la enfermedad incluye la ECJ, kuru, insomnio fatal familiar (IFF), síndrome de Gerstmann-Straussler-Scheinker (GSS) y la nueva variante de la ECJ (nvCJD). Entre los animales, se destaca la encefalopatía espongiforme bovina (EEB) y el scrapie, entre las ovejas ${ }^{1}$.

Correspondencia a: Dr. Luis Cartier R. Luis Thayer Ojeda 717. E mail: lcartier@med.uchile.cl
La ECJ es una encefalopatía invariablemente mortal, que anualmente mata en el mundo a una persona por cada millón de habitantes, pero en Chile afecta a dos o más personas por millón ${ }^{2-3}$. La ECJ suele desarrollarse entre la quinta y sexta década de la vida, aunque hay formas que pueden iniciarse más precozmente ${ }^{4}$. La expresión clínica puede ser variada en el comienzo de la enfermedad, generando dificultades diagnósticas. Sin embargo, esta encefalopatía siempre converge hacia un cuadro demencial subagudo, acompañado de síntomas motores (piramidales, cerebelosos, extrapiramidales) y visuales, así como de mioclonías. La sobrevida no suele superar el $a \tilde{n} 0^{5}$. Tiene como parámetros 
diagnósticos, además de los aspectos clínicos, el electroencefalograma (EEG) que suele presentar una actividad espicular pseudoperiódica característica ${ }^{6}$; la resonancia magnética (RM), que con técnicas de difusión, puede mostrar hiperintensidades en los núcleos estriados (putamen y caudado) y en áreas de la corteza cerebral ${ }^{7}$. El examen citoquímico estándar del líquido cefalorraquídeo (LCR) suele ser normal, aunque presenta aumento de la proteína 143-3-8, así como de la metaloproteinasa MMP-99. Finalmente, el estudio histopatológico del cerebro puede establecer la encefalopatía espongiforme y eventualmente la presencia de la proteína prión en el sistema nerviosos central (SNC).

De acuerdo a su origen, la ECJ ha sido clasificada en esporádica, familiar e iatrogénica. La forma esporádica es la más frecuente, constituye casi $90 \%$ de los casos, la forma familiar da cuenta de $10 \%$ y la iatrogénica, que se relaciona a la acción contaminante de tejidos (trasplante de córnea u otros), representa menos de $1 \%^{2}$.

Todas las expresiones clínicas de la enfermedad de Creutzfeldt-Jakob tienen en común una encefalopatía espongiforme, que se asocia a la presencia, en el tejido cerebral, de una proteína malformada, que tiende a polimerizar y a depositarse como amiloide. Esta proteína anómala, fue denominada prión por Prusinier ${ }^{10}$. La proteína prión o $\mathrm{PrPSC}^{\mathrm{Sc}}$, es la isoforma anormal de la proteína natural $\mathrm{PrPC}^{\mathrm{C}}$, que tiene idéntica secuencia aminoacídica, pero distinta disposición espacial. Condición que concede a la proteína prión la capacidad de autorreplicarse y modificar su comportamiento bioquímico. Mientras el prión $\mathrm{PrPSc}$ es insoluble y posee resistencia al tratamiento con la proteinasa $\mathrm{K}$; la proteína $\mathrm{PrPc}^{\mathrm{C}}$ es soluble y susceptible a la proteolisis $^{10}$. La diferencia estructural terciaria de ambas proteínas, está basada en la conformación de sus plegamientos, la proteína natural $\mathrm{PrP}^{\mathrm{C}}$ tiene predominio de estructuras del tipo $\alpha$-hélices; mientras que la proteína prión $\mathrm{PrPS}^{\mathrm{Sc}}$ muestra una mayor cantidad de láminas tipo $\beta$, en su plegamiento ${ }^{11}$.

La proteína $\mathrm{PrP}^{\mathrm{C}}$ tiende a disponerse en las regiones sinápticas de las neuronas, esa condición y la aberrante capacidad de $\mathrm{PrPSc}$ para trasformar la normal $\mathrm{PrPc}^{\mathrm{C}}$ en un isómero patológico, estarían en el origen de la encefalopatía espongiforme ${ }^{12-13}$. La necesaria presencia de la proteína normal para desarrollar la enfermedad, se ha puesto en evidencia con ratones transgénicos que tienen un knock- out del gen de la proteína $\operatorname{PrPc}$, que en el hombre se localiza en el brazo corto de cromosoma 20. La administración experimental de priones, en estos ratones transgénicos, no logra el desarrollo de la encefalopatía. La reintroducción del gen de la PrPc, los vuelve susceptibles a la enfermedad ${ }^{14}$. Aparentemente, la acumulación de $\mathrm{PrPSc}$ sería altamente deletérea para las células; hecho que llevaría a generar cambios en las áreas sinápticas de las neuronas, ubicación preferencial de la espongiosis. La PrPSc es una proteína de membrana, insoluble en su fragmento $\operatorname{PrP}$ 27-30, que tiende a acumular$\mathrm{se}^{15}$. El tejido cerebral pareciera ser la única estructura disponible para la replicación de la proteína prión, tanto en las formas esporádicas, como familiares de la ECJ. En estas últimas, la replicación de la proteína malformada, podría verse facilitada por la mutación en el codón 200, que posiblemente aumenta la inestabilidad conformacional de la proteína $\mathrm{PrP}^{\mathrm{C}}$ en estos sujetos ${ }^{16}$.

Se ha establecido que el fenotipo clínico de la ECJ, ya sea esporádica o familiar, se relaciona con el genotipo del individuo afectado. Se han definido 6 formas clínicas, que se han relacionado con los genotipos posibles del codón 129. Si este codón es homocigoto (Met/Met), (Val/Val) o heterocigoto (Met/Val), genera proteínas prión distintas, que además pueden ser largas (tipo I) o cortas (tipo II), lo que permitiría distinguir al menos seis genotipos. Los distintos genotipos parecieran condicionar la capacidad de los priones para iniciar el cambio estructural en determinadas áreas cerebrales, dando origen a las distintas formas clínicas descritas ${ }^{17}$.

En consideración a la elevada incidencia de la ECJ en Chile y la inusual frecuencia de formas familiares ${ }^{18-20}$, se decidió extender el análisis de las condiciones de susceptibilidad a miembros de familias chilenas afectadas por la ECJ. Se estudió la presencia de marcadores genéticos en 50 miembros de cuatro familias, identificando las mutaciones o genotipos que, de acuerdo a lo aceptado internacionalmente, condicionarían la capacidad para enfermar.

\section{MATERIAL Y MÉTODO}

Pacientes. Los casos índices de cada familia, que tenían el antecedente familiar de enfermedad de Creutzfeldt-Jakob y en los que se hizo el diagnósti- 
co de la enfermedad por el cuadro clínico y exámenes complementarios, fueron la base del estudio. En ellos se definió una demencia subaguda, asociada inicialmente a temblor de las extremidades superiores, disartria e inestabilidad de la marcha, signos piramidales, cerebelosos y alteraciones visuales, según cada caso, que terminaron en mutismo akinético. El diagnóstico clínico se asoció con el hallazgo de actividad espincular periódica en el EEG; la presencia de zonas hiperintensas en la corteza y ganglios basales del cerebro, en la $\mathrm{RNM}$, y se corroboró con un aumento significativo de la proteína 14-3-3 en el LCR. Además del antecedente cierto de parientes fallecidos con el mismo diagnóstico, como se observa en el árbol genealógico de cada familia (Figuras 1, 2, 3 y 4). En dos familias se dispuso del diagnóstico anatomopatológico en uno de sus miembros. Todas las personas que participaron en este estudio lo hicieron aceptando previamente un consentimiento informado, de acuerdo a las normas definidas por el Comité de Ética del Hospital del Salvador, y la mayoría de ellos manifestaron un interés personal en incorporarse al estudio. Aunque varios miembros de distintas familias no lo aceptaron.

Muestras. En cada enfermo índice se tomaron 10 $\mathrm{ml}$ de sangre y $3 \mathrm{ml}$ de LCR, que permitió iniciar el estudio de cada grupo familiar. Una vez establecido el diagnóstico de ECJ familiar, se tomó $3 \mathrm{ml}$ de sangre a cada uno de los parientes interesados en participar en el estudio y que aceptaron el Consentimiento Informado.

Amplificación de ADN por PCR. Las células mononucleares de la sangre (peripheral bloodmononuclear cells [PBMCs]) de cada muestra fueron purificadas por centrifugación en gradientes de Ficoll-Hypaque. El ADN de los PBMCs fue extraído por lisis de las células usando la resina Chelex 100. La reacción en cadena de la polimerasa fue realizada con los partidores, CJD-A5'actgagaattctctgacattctcctcttca3' y CJD-B 5 'tactgaggatccctcaagctggaaaacaggaagacc $3^{\prime 21}$. La condición de PCR consistió en una denaturación inicial de 5 min a $94^{\circ} \mathrm{C}$ y 35 ciclos, $^{\circ} 4^{\circ} \mathrm{C}$ por $1 \mathrm{~min}$, $57^{\circ} \mathrm{C}$ por $1 \mathrm{~min}$ y $72^{\circ} \mathrm{C}$ por $1 \mathrm{~min}$.

Secuenciamiento directo por PCR. Secuenciamiento de ambas hebras de ADN del gen que codifica para la proteína PrP, fue realizado con un kit de secuenciamiento directo de ABI PRISM (Applied Biosystems, Foster, USA) que contiene la enzima AmpliTaq ADN polimerasa. Las mezclas de reacción de secuenciamiento fueron fraccionadas y analizadas en un secuenciador automático de ADN (ABI PRISM modelo 310; Applied Biosystems, Foster, USA). Los siguientes partidores fueron utilizados para el secuenciamiento: A 5'actgagaattctctgacattctcctcttca 3', B 5’tactgaggatccctcaagctggaaaacaggaagacc 3' y K-5 5'CATGAGCAGGCCCATCATAC 3', K-6 5TGTGTAGACGAGTTGGTGCGCG 3' descritos previamente ${ }^{22}$.

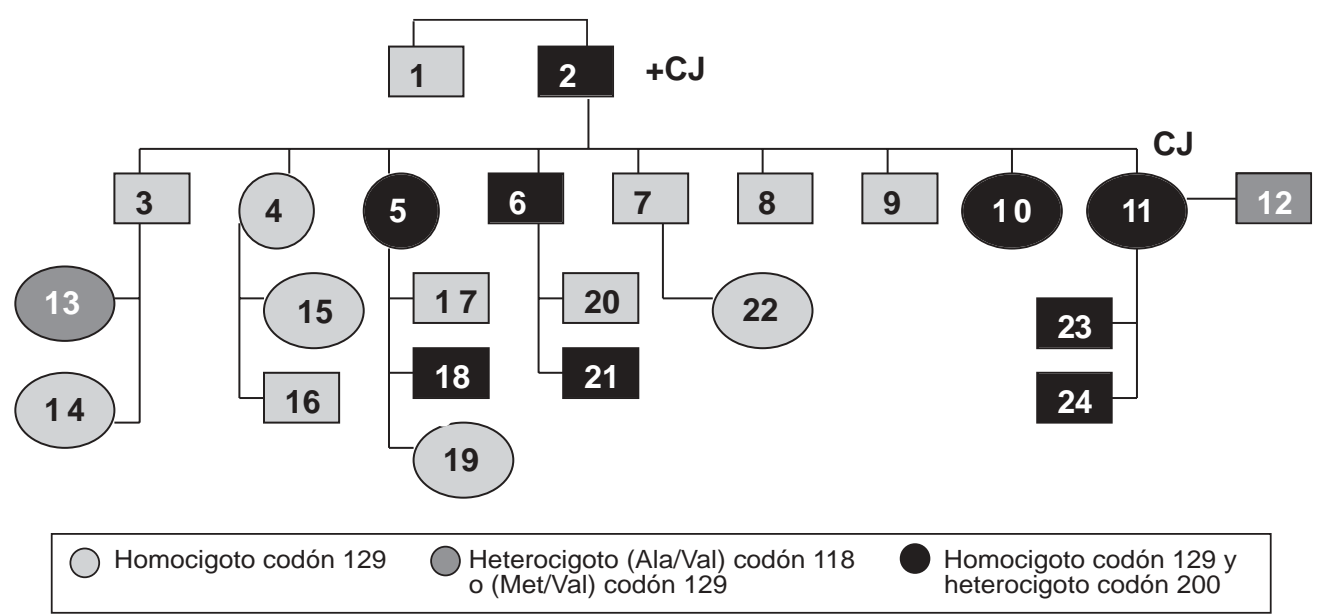

Figura 1. Árbol genealógico familia A mutaciones genéticas de susceptibilidad para la ECJ.

Paciente 11: Caso índice; Paciente 2: sin muestra, problablemente homocigoto codón 129 (Met/Met) y heterocigoto (Glu/Lys) codón 200. 


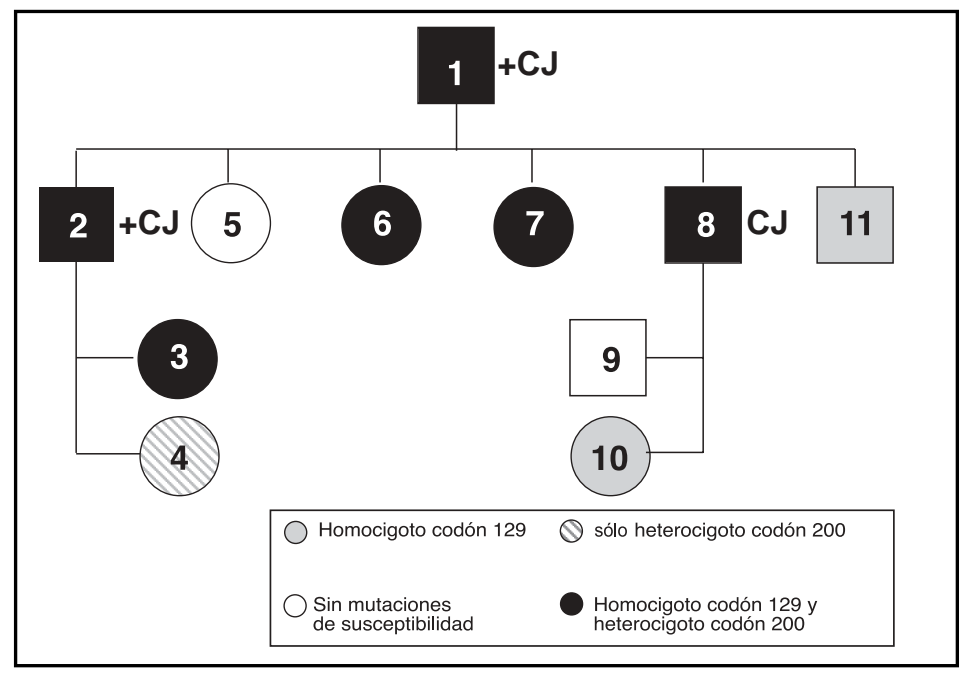

Figura 2. Árbol genealógico familia B mutaciones genéticas de susceptibilidad para la ECJ.

Paciente 8: Caso índice; Paciente 1 y 2: sin muestra, fallecieron de CJD, probablemente homocigotos (Met/Met) codón 129 y heterocigoto (Glu/Lys) codón 200.

Figura 3. Árbol genealógico familia $\mathrm{C}$ mutaciones genéticas de susceptibilidad para la ECJ. Paciente 3: Caso índice; Paciente 1 y 4: sin muestra, fallecieron de CJD, probablemente homocigotos (Met/Met) codón 129 y heterocigoto (Glu/Lys) codón 200.
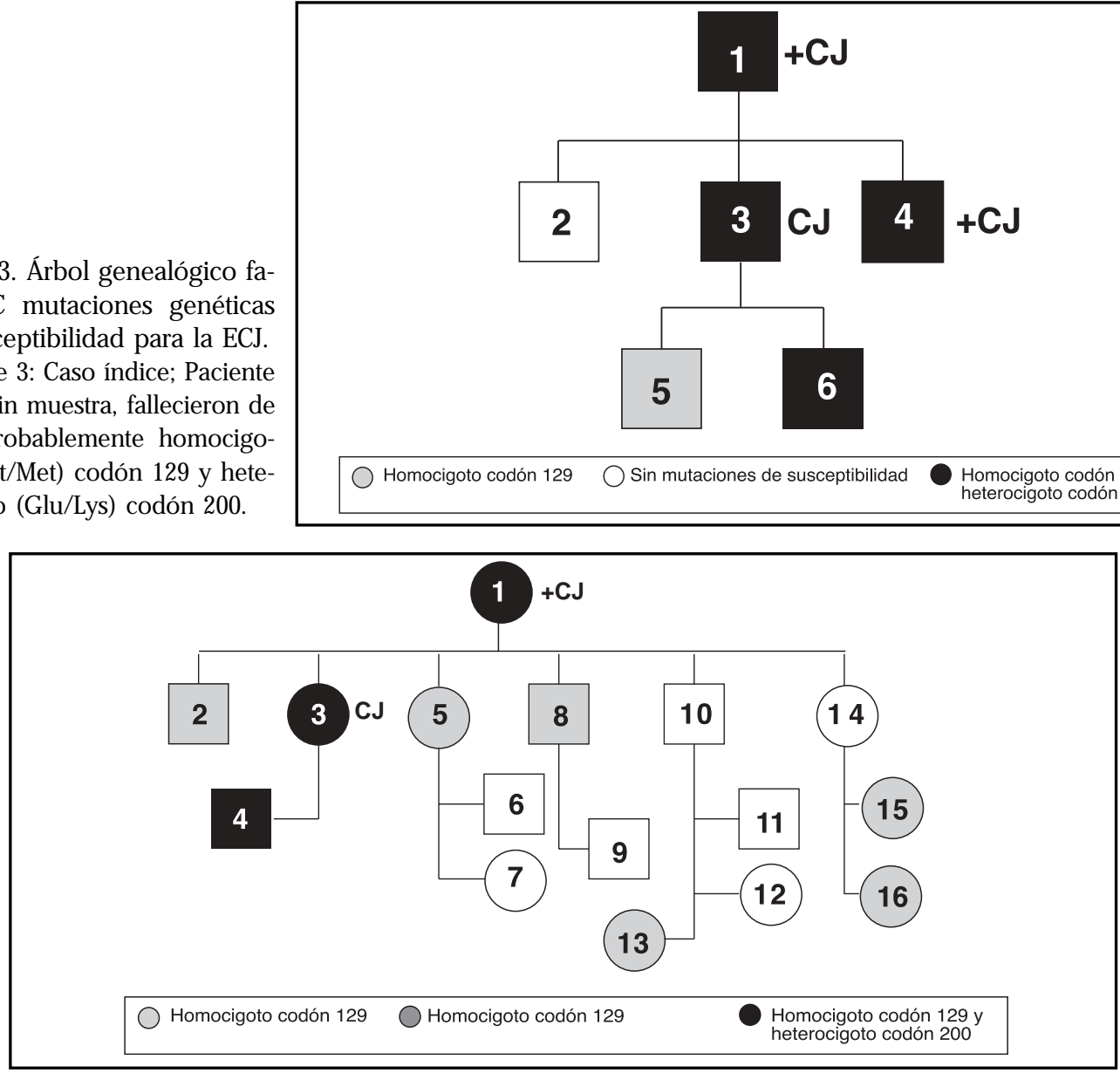

Figura 4. Árbol genealógico familia D mutaciones genéticas de susceptibilidad para la ECJ.

Paciente 3: Caso índice; Paciente 1: sin muestra, falleció de CJD, probablemente homocigoto (Met/Met) codón 129 y heterocigoto (Glu/Lys) codón 200; Paciente 14: sin muestra, probablemente sin mutaciones de susceptibilidad. 


\section{RESULTADOS}

Se estudió la incidencia de marcadores genéticos de susceptibilidad para adquirir la ECJ, en 50 miembros de cuatro familias, repartidos en tres generaciones. Se identificaron los genotipos o mutaciones que han sido sugeridos como predisponentes para desarmollar la enfermedad. En la familia A integrada por 24 miembros, sólo en 23 se obtuvo muestras, el otro integrante corresponde al padre del caso índice fallecido de ECJ. En esta familia se encontraron 21 miembros homocigotos (Met/Met) para el codón 129, ocho de los cuales fueron simultáneamente heterocigotos (Glu/Lys) en el codón 200 (Figura 1). Además, se encontró un miembro (sujeto 12) heterocigoto (Met/Val) en el codón 129 y otro (sujeto 13) heterocigoto (Ala/Val) en el codón 118; esta última mutación no está reportada de conferir susceptibilidad para la ECJ. En la segunda generación, 4 de 9 miembros tuvieron las dos mutaciones simultáneamente. En la tercera generación 4 de 7 miembros de esta descendencia tenían mutación en los codones 129 y 200, hechos que sugiere una selección positiva del genotipo doble mutante en esta familia. Sin embargo, de los 23 miembros estudiados, sólo uno de ellos con la doble mutación en los codones 129 y 200 (sujeto 11) presentó síntomas de ECJ (caso índice). Este paciente transmitió la doble mutación a dos hijos en forma dominante. Suponemos que el abuelo (sujeto 1) tenía la doble mutación.

En la familia B, compuesta de 11 miembros, no se estudiaron genéticamente 2 casos fallecidos previamente de la ECJ, el padre (sujeto 1) y un hermano del caso índice (sujeto 2). En esta familia, 6 de 9 miembros fueron homocigotos (Met/Met) en el codón 129, cuatro simultáneamente heterocigotos (Glu/Lys) para el codón 200 y un sujeto, únicamente heterocigoto (Glu/ Lys) en el codón 200 (Figura 2). El caso índice (sujeto 8) desarmollaba la enfermedad en el momento de iniciar el estudio. En la segunda generación, 3 de 5 miembros presentaban las dos mutaciones simultáneamente, y suponemos que los miembros fallecidos de la ECJ, también tenían ambas mutaciones. En la tercera generación, en 1 de 4 miembros examinados, se encontró la doble mutación, en 2 sólo se detectó una de ambas mutaciones (sujetos 4 y 10). De los 11 miembros de esta familia, 3 han sufrido la enfermedad.

La familia $C$ compuesta por 6 miembros, en 4 se obtuvo muestra para el secuenciamiento del gen de la proteína PrPC. Los otros dos, fallecidos de la ECJ son el padre (sujeto 1), que tiene anatomía patológica confirmatoria y un hermano del caso índice (sujeto 4). En esta familia todos los integrantes estudiados eran homocigotos (Met/Met) en el codón 129 y 2 simultáneamente heterocigotos (Glu/Lys) en el codón 200 (Figura 3). En la segunda generación, 1 de 2 miembros estudiados, tenía las dos mutaciones simultáneamente y comesponde al caso índice (sujeto 3) el otro era homocigoto (Met/Met) en el codón 129, el tercer miembro de esta generación falleció de la ECJ y presumiblemente tenía un genotipo con la doble mutación. Uno de los hijos del caso índice también mostró la doble mutación y otro fue homocigoto (Met/ Met) en el codón 129. De 6 sujetos de esta generación, tres presentaron la ECJ (individuos 1, 3 y 4), sin mutaciones de susceptibilidad sólo uno (sujeto 2).

La familia D, integrada por 16 miembros, sólo en 14 se obtuvo muestra para el secuenciamiento del gen de la proteína PrPC. La madre del caso índice (sujeto 1), falleció de la ECJ, confirmada con anatomía patológica. En esta familia se encontró que 8 de 14 integrantes eran homocigotos (Met/Met) en el codón 129, y 2 simultáneamente heterocigotos (Glu/Lys) en el codón 200 (Figura 4). La segunda generación mostró en 1 de 6 miembros las dos mutaciones en los codones 129 y 200, que comesponde al caso índice (sujeto 3); homocigosis (Met/Met) en el codón 129 se observó en 3 individuos y 2 no mostraron cambios genéticos de susceptibilidad. De 9 miembros de la tercera generación, sólo 1 mostró la doble mutación (sujeto 4), hijo del sujeto con doble mutación de la generación anterior; en 3 hubo homocigosis en el codón 129 y 5 no tenían mutaciones de susceptibilidad para la ECJ. De 16 parientes de este grupo familiar, dos presentaron la enfermedad (sujetos 1 y 3 ).

En resumen, las cuatro familias estaban constituidas por 57 miembros, se dispuso de muestras en 50 de ellos. De éstos, 40 presentaron algún cambio en el genotipo que sugenía susceptibilidad para desarrollar la ECJ. Veintitrés sujetos sólo eran homocigotos (Met/Met) en el codón 129; 16 presentaban simultáneamente mutaciones tanto en el codón 129 como 200 y 1 fue exclusivamente heterocigoto (Glu/Lys) en el codón 200 (Tabla 1). Dos sujetos presentaron mutaciones no asociables a susceptibilidad, y en 8 no se detectaron mutaciones genéticas de susceptibilidad. Ninguno de los sujetos estudiados mostró polimorfismo en el codón 219. Adicionalmente y considerando a todos los integrantes de los 4 grupos familiares, diez habían contraído la ECJ (17,5\%). 


\section{DisCUSIÓN}

Se estima que en nuestro país cada año fallecen 40 personas por la ECJ, y que Chile muestra la más alta incidencia a nivel mundial de la forma familiar de la ECJ ${ }^{3}$. Por ello, en esta investigación nos orientamos al análisis de 4 familias chilenas afectadas por la ECJ. Secuenciamos el genotipo de la proteína $\operatorname{PrP}$ en 50 miembros de estas diferentes familias, e identificamos aquellas mutaciones 0 genotipos que han sido descritos como predisponentes para el desarrollo de la ECJ ${ }^{16,17}$.

En este estudio pudimos establecer que 32\% (16/50) de los sujetos analizados presentaban una mutación doble, en los codones 129 y 200, que definían un genotipo predisponente para contraer la ECJF. Asimismo, en 80\% (40/50) de los sujetos, se observó al menos una mutación de susceptibilidad para desarrollar la ECJ, haciendo evidente también, una tendencia a la transmisión dominante de los genotipos predisponentes. Estos hallazgos harían comprensible la inusual alta incidencia de la ECJ familiar y esporádica en Chile. Además, todos los casos índices de cada familia presentaban la doble mutación genómica, así como la transmisión dominante del genotipo en cada caso.

Sin embargo, la mayor incidencia de la enfermedad no parece estar vinculada únicamente a factores genéticos. También pareciera necesaria la participación de otras condiciones aún no definidas, para el desarrollo de la ECJ. Si bien 17,5\% (10/57) de esta serie presentó la ECJ, existe un numeroso grupo de estos sujetos que permanece asintomático, a pesar de ser portadores de los genotipos predisponentes. El hecho genético aclaranía la mayor incidencia de la ECJ familiar en Chile, pero no explicanía por qué sujetos susceptibles y con edad para enfermar, no hayan desarmollado la enfermedad.

Conocemos los factores que son predisponentes para iniciar la enfermedad. Sin embargo, no hay estudios, respecto de condiciones que pudiesen ser protectoras, excepto la publicación de Shibuya, quien observó que la presencia de polimorfismo en el codón 219 Lys (cambio de glutamina por lisina), protegería de la enfermedad ${ }^{23}$; genotipo que no fue encontrado entre nosotros. Tampoco se conoce el modo natural de la transmisión de los priones entre individuos; ni se han establecido los mecanismos endógenos que pudiesen desencadenar, en sujetos susceptibles, el plegamiento anormal de la $\mathrm{PrP}^{\mathrm{C}}$. Asimismo, no se han analizado factores ambientales que pudieran intervenir o contribuir en la activación de plegamientos anormales de la proteína PrP.

Las cuatro incógnitas relativas a: factores protectores, modo de transmisión natural, participación de mecanismos endógenos y factores ambientales, deben ser temas de estudio en Chile. Considerando que tenemos los índices más altos de la ECJ en el mundo, a ello debiera agregarse el desamollo de procedimientos para el diagnóstico precoz de la enfermedad y búsquedas de alcance terapéutico. En estas condiciones, es muy razonable proponer para Chile el desarmollo de un programa nacional de vigilancia clínico-epidemiológico de los casos sospechosos de ECJ. Ello permitináa investigar adecuadamente esta enfermedad, que anualmente tiene una morbimortalidad superior al de ciertas virosis de impacto nacional. La instauración de un programa de vigilancia nacional de la ECJ está al alcance de nuestro país, ya que se dispone de métodos moleculares, para evaluar la susceptibilidad genética de los sujetos. Además, hallazgos recientes han permitido amplificar pequeñas

Tabla 1. Resumen de las mutaciones observadas en el gen de la proteína PrPc en cincuenta miembros de cuatro familias chilenas portadoras de la ECJF

\begin{tabular}{|lrccccc|}
\hline Familia & $\mathrm{n}$ & $\begin{array}{c}129 \\
\text { Met/Met }\end{array}$ & $\begin{array}{c}200 \\
\text { Glu/Lys }\end{array}$ & $\begin{array}{c}129+200 \\
\text { Met/Met } \\
\text { Glu/Lys }\end{array}$ & $\begin{array}{c}129 \\
\text { Met/Val }\end{array}$ & $\begin{array}{c}129+118 \\
\text { Met/Val }\end{array}$ \\
\hline A & 23 & 13 & 0 & 8 & 1 & 1 \\
B & 9 & 2 & 1 & 4 & 2 & 0 \\
C & 4 & 2 & 0 & 2 & 0 & 0 \\
D & 14 & 6 & 0 & 2 & 6 & 1 \\
Total & 50 & 23 & 1 & 16 & 9 & 0 \\
\hline
\end{tabular}

n: número de integrantes con muestras y secuencia genética del ADN. 
cantidades de la proteína prón, obtenidas de tejido nervioso o de la sangre. Ello genera buenos augurios

\section{REFERENCIAS}

1. Cownge J. Molecular neurology of prion disease. J Neurol Neurosurg Psychiatry 2005; 76: 906-19.

2. Johnson RT, GibBs CJ JR. Creutzfeldt-Jakob disease and related transmissible spongiform encephalopathy. N Engl J Med 1998; 339: 1994-2004.

3. GAJDUSEK DC. Infectious Amyloids: Subacute Spongiform Encephalopathies as Transmisible Cerebral Amyloidoses. In: Fields BN, Knipe DM, Howley PM y cols, ed. Fields Virology. Filadelfia: Editorial Lippincott-Raven Publishers, 1996; 2851-900.

4. WiL RG, IRonside JW, Zeidier M, Cousens SN, Estibeiro K, ALPEROVITCH A ET AL. A new variant of CreutzfeldtJakob disease in the UK. Lancet 1996; 347: 921-5.

5. Gálvez S, Cartier L Análisis clínico de una serie de 69 casos definitivos de la enfermedad de Creutzfeldt-Jakob ocurridos en Chile entre 19601985. Rev Méd Chile 1987; 115; 1148-54.

6. Chiofalo N, Fuentes A, Gálvez S. Serial EEG findings in 27 case of Creutzfeldt-Jakob disease. Arch Neuro l 1980; 37: 143-5.

7. Demaerel P, Sciot R, Robberecht W, Dom R, VanderMEULEN D, MAES F ET AL. Accuracy of diffusionweighted MR imaging in the diagnosis of sporadic Creutzfeldt-Jakob disease. J Neurol 2003; 250: 222-5.

8. Beaudry P, Cohen P, Brandl JP, Deiasnerie-Laupretre N, Richard S, Launay JM et al 14-3-3 protein, neuronspecific enolase, and S-100 protein in cerebrospinal fluid of patients with Creutzfeldt-Jakob disease. Dement Geriatr Cogn Disord 1999; 10: 40-6.

9. Kettlun A, Collados L, García L, Cartier L, Wolf ME, MosNaim AD ET AL. Matrix metalloproteinase profile in patients with Creuztfeldt-Jakob disease. Int J Clin Pract 2003; 57: 475-8.

10. Prusiner SB. Prions. Proc Natl Acad Sci USA 1998; 95: 13363-83.

11. Pan KM, Balwin M, Nguyen J, Gasset M, Serban A, GROTH D ET AL Conversion of alpha-helices into betasheets features in the formation of the scrapie prion proteins. Proc Natl Acad Sci USA 1993; 90: 10962-6.

12. Kocisko DA, Come JH, Priola SA, Chesebro B, Raymond GJ, LANSBURY PT ET AL Cell-free formation of proteaseresistant prion protein. Nature 1994; 370: 471-4.

13. SоTO C, SABORIO GP, ANDERES L Cyclic amplification of protein misfolding: application to prion-related disorders and beyond. Trends Neurosci 2002; 25: 390-4. en la posibilidad de un diagnóstico presintomático, en estas formas familiares de la enfermedad ${ }^{24,25}$.

14. Legname G, Baskakov IV, Nguyen HO, Riesner D, Cohen FE, Dearmond SJ ET al. Synthetic mammalian prions. Science 2004; 305: 673-6.

15. Cohen FE, Prusiner SB. Pathologic conformations of prion proteins. Annu Rev Biochem 1998; 67: 793-819.

16. Goldfard LG, Brown P, Mitrova E, Cervenakova L, Goldin L, Korczyn AD ET AL. Creutzfeldt-Jacob disease associated with the PRNP codon 200 Lys mutation: an analysis of 45 families. Eur J Epidemiol 1991; 7: 477-86.

17. Parchi P, Giese A, Capelari S, Brown P, SchulzSCHAEFFER W, WindL O ET aL. Classification of sporadic Creutzfeldt-Jakob disease based on molecular and phenotypic analysis of 300 subjects. Ann Neurol 1999; 46: 224-33.

18. Brown P, Gálvez S, Goldfarb Lg, Nieto A, Cartier L, GibBs CJ JR ET aL. Familial Creutzfeldt-Jakob disease in Chile is associated with the codon 200 mutation of the PRNP amyloid precursor gene on chromosome 20. J Neurol Sci 1992; 112: 65-7.

19. Cartier L, Gálvez S, Gajdusek DC. Familial clustering of the ataxic form of Creutzfeldt-Jakob disease with Hirano bodies. J Neurol Neurosurg Psychiatry 1985; 48: 234-8.

20. Gálvez S, Cartier L, Monari M, Araya T. Familial Creutzfeldt-Jakob in Chile. J Neurol Sci 1983; 59: 139-47.

21. Palmer MS, Dryden AJ, Hughes JT, Cownge J. Homozygous prion protein genotype predisposes to sporadic Creutzfeldt-Jakob disease. Nature 1991; 352: 340-2.

22. Jeong BH, Ju WK, Huh K, Lee EA, Choi IS, Im JH et AL. Molecular analysis of prion protein gene (PRNP) in Korean patients with Creutzfeldt-Jakob disease. J Korean Med Sci 1998; 13: 234-40.

23. Shibuya S, Higuchi J, Shin RW, Tateishi J, Kitamoto T. Codon 219 Lys allele of PRNP is not found in sporadic Creutzfeldt-Jakob disease. Ann Neurol 1998; 43: 826-8.

24. CAStiLa J, SaA P, Hetz C, Soto C. In vitro generation of infectious scrapie prions. Cell 2005; 121: 195-206.

25. Soto C, Anderes L, Suardi S, Cardone F, Castila J, Frossard MJ et al. Pre-symptomatic detection of prions by cyclic amplification of protein misfolding. FEBS Lett 2005; 579: 638-42. 